\title{
CONFORMAL MODULUS AND PLANAR DOMAINS WITH STRONG SINGULARITIES AND CUSPS*
}

\author{
HARRI HAKULA ${ }^{\dagger}$, ANTTI RASILA $^{\ddagger}$, AND MATTI VUORINEN $§$
}

\begin{abstract}
We study the problem of computing the conformal modulus of rings and quadrilaterals with strong singularities and cusps at their boundary. We reduce this problem to the numerical solution of the associated Dirichlet and Dirichlet-Neumann-type boundary values problems for the Laplace equation. Several experimental results, with error estimates, are reported. In particular, we consider domains with dendrite-like boundaries where an analytic formula for the conformal modulus can be derived. The boundary value problems are solved using an $h p$-finite element method.
\end{abstract}

Key words. conformal capacity, conformal modulus, quadrilateral modulus, $h p$-FEM, numerical conformal mapping

AMS subject classifications. 65E05, 31A15, 30C85

1. Introduction. The conformal modulus is an important tool in geometric function theory [1]. It is defined as the Dirichlet energy or $H^{1}$-seminorm of the Laplacian under certain boundary conditions and thus closely related to certain physical quantities which also occur in engineering applications such as resistance values of integrated circuit networks; see, e.g., $[22,24]$.

Consider a rectangle with vertices $(0,0),(1,0),(1, h),(0, h)$ and solve first $-\Delta u_{1}=0$ with $u_{1}=1$ along the edge $(0,0),(1,0)$ and $u_{1}=0$ along the edge $(1, h),(0, h)$, and $\partial u_{1} / \partial n=0$ otherwise, and second, the conjugate problem, $-\Delta u_{2}=0$ with the Dirichlet and Neumann conditions interchanged along the edges. It is clear that the modulus of the quadrilateral corresponding to $u_{1}$ is $R_{1}=h$ and that of the conjugate problem $R_{2}=1 / h$, with the reciprocal identity $R_{1} R_{2}=1$. Remarkably, this identity holds for all simply connected domains since by definition they can be mapped onto such a rectangle. Notice that for symmetric cases, the conformal modulus is identically equal to one.

For instance, the famous L-shaped domain is defined with six vertices. The construction above states that any four vertices can be chosen to divide the boundary into Dirichlet and Neumann parts. In Figure 1.1 an example of such an L-shaped configuration is given with the numerically computed moduli up to machine precision (the Dirichlet boundaries are indicated with dashed lines in Figure 1.1a). We arrive at two important observations: first, the errors of the $H^{1}$-seminorms of the resulting potentials can be estimated reliably without any a priori knowledge of the exact potential, and second, the quality of any 2D discretization used in the numerical solution of a PDE can be assessed using the reciprocal relation—an especially useful result when the boundary is not smooth.

We consider both simply and doubly connected bounded domains. By definition such a domain can be mapped conformally either onto a rectangle or onto an annulus, respectively. For the numerical study of these two cases, we define the modulus $h$ as follows. In the simply connected case, as outlined above, we fix four points on the boundary of the domain, call a domain with these fixed boundary points a quadrilateral, and require that these four points are

*Received November 11, 2016. Accepted November 8, 2018. Published online on January 8, 2019. Recommended by Tom Delillo.

${ }^{\dagger}$ Aalto University, Institute of Mathematics, P.O. Box 11100, FI-00076 Aalto, Finland (harri.hakula@aalto.fi).

${ }^{\ddagger}$ Guangdong Technion-Israel Institute of Technology, College of Science, 241 Daxue Road, Shantou, Guangdong 515063, China (antti.rasila@iki.fi; antti.rasila@gtiit.edu.cn).

${ }^{\S}$ Department of Mathematics and Statistics, FI-20014 University of Turku, Finland (vuorinen@utu.fi). 


\section{ETNA}

Kent State University and Johann Radon Institute (RICAM)

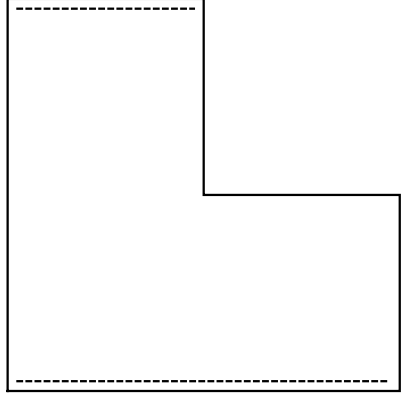

a) Domain with Dirichlet boundaries indicated.

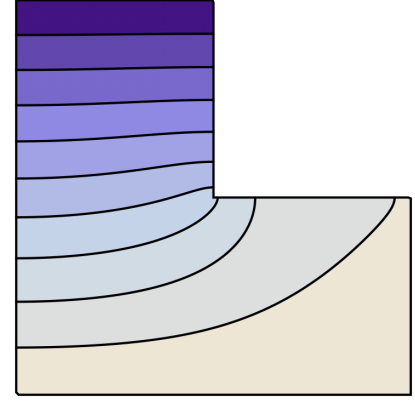

b) Potential function.

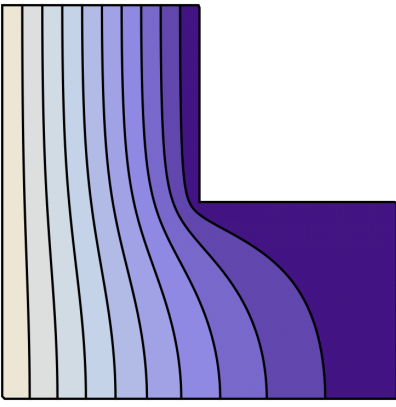

c) Potential function of the conjugate problem.

FIG. 1.1. Conformal modulus: unit L-shaped domain. $R_{1}=0.5773502691896245, R_{2}=1.7320508075688785 ; R_{1} R_{2}=1$.

mapped onto the vertices $(0,0),(1,0),(1, h),(0, h)$ of the rectangle. In the doubly connected case we require that the annulus is $\left\{(x, y): \exp (-h)<x^{2}+y^{2}<1\right\}$. Doubly connected domains are also called ring domains or simply rings. Surveys of state of the art methodologies in the field are presented in the recent books by N. Papamichael and N. Stylianopoulos [22] and by T. Driscoll and L. N. Trefethen [28]. Various applications are described in [16, 17, 24, 29]. In the past few years, quadrilaterals and ring domains of increasing complexity have been studied by several authors $[5,6,10,23,27]$.

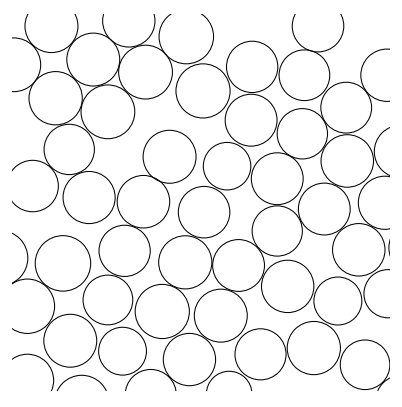

a) Carbon fibre composite geometry. Image data courtesy of I.Babuška/UT Austin.

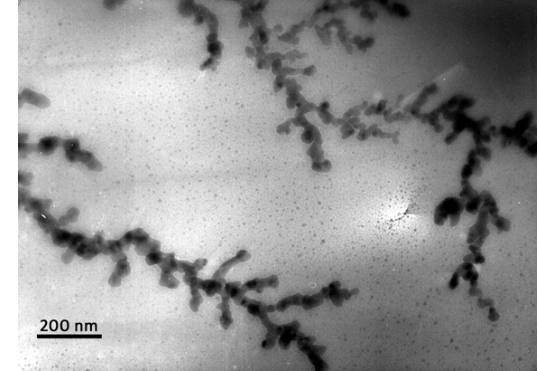

b) Dendritic growth. Image courtesy of G.M. Stone/UC Berkeley and LBNL.

FIG. 1.2. Examples of realistic domains with cusps and strong singularities.

Many interesting applications involving novel designs with elaborate boundaries with cusps and singularities have emerged. In this paper we present both benchmark problems and a computational methodology for evaluating and ensuring proper discretizations for the numerical simulations of such problems. Some specific application classes are: (with cusps) the modeling of carbon fibre composites [4] and conformal mappings in the study of metamaterials $[3,26]$, (dendrite boundaries) the dendritic growth in two-metal electrodeposition-a primary failure mechanism in many battery chemistries [20,21] — and self-similar structures in fractal antenna designs [18]. We emphasize that the citations are only representative and do not cover the whole fields.

In the study of metamaterials one of the interesting configurations is where two domains 
with different material properties have minimal interfaces, in other words, with cusps in the domain. Similarly, in fibre composites, cusps form naturally when two fibres are in contact; see Figure 1.2a). Recently, Bergweiler and Eremenko [8] have studied quadrilaterals with characteristics of precisely this type. We use their work as a foundation and compute the canonical conformal mappings numerically using the algorithm presented in [13].

The fractal antenna design problem belongs to the class of ring problems of dendrite type (i.e., continua without loops) [30]. Of course, it is also interesting to compute the effect on the capacity of dendritic growth in capacitors; see Figure 1.2b). As the main new result of this paper, a new class of ring domains is introduced. This class of ring domains of dendrite type is characterized by a triplet of parameters $(r, m, p)$, its construction is recursive, and yet its conformal modulus can be explicitly given. By varying the parameter values or the recursion level of the construction, one can increase the computational challenge, and therefore, this family of domains forms a valuable set of test problems. Using standard techniques, the ring problem can be reinterpreted as a quadrilateral one, and thus, the reciprocal error estimate is also available.

Our numerical solution method of choice is the $h p$-finite element method (FEM) as implemented in [14]. Through our experiments we relate the reciprocal error estimate to known $h p$-error estimation techniques, in particular, to the auxiliary subspace error estimation (often called hierarchic error estimation), and show numerically that the convergence rates are similar with constants depending on the domain aspect ratios.

The paper is organized as follows: in Section 2 the definitions of the basic concepts are given. Domains with cusps are discussed in Section 3 with a detailed numerical study of the reciprocal error and its relation to standard error estimates. The dendrite problem is the focus of Section 4. Finally, conclusions are drawn in Section 5.

2. Preliminaries. In this section central concepts to our discussion are introduced. The quantities of interest from function theory are related to numerical methods, and the error estimators arising from the basic principles are defined.

2.1. The conformal modulus. A simply connected domain $D$ in the complex plane $\mathbb{C}$ whose boundary is homeomorphic to the unit circle is called a Jordan domain. A Jordan domain $D$, together with four distinct points $z_{1}, z_{2}, z_{3}, z_{4}$ in $\partial D$, which occur in this order when traversing the boundary in the positive direction, is called a quadrilateral and denoted by $\left(D_{1} ; z_{1}, z_{2}, z_{3}, z_{4}\right)$. If $f: D \rightarrow f D$ is a conformal mapping onto a Jordan domain $f D$, then $f$ has a homeomorphic extension to the closure $\bar{D}$ (also denoted by $f$ ). We say that the conformal modulus of $\left(D ; z_{1}, z_{2}, z_{3}, z_{4}\right)$ is equal to $h>0$ if there exists a conformal mapping $f$ of $\bar{D}$ onto the rectangle $[0,1] \times[0, h]$, with $f\left(z_{1}\right)=1+i h, f\left(z_{2}\right)=i h, f\left(z_{3}\right)=0$, and $f\left(z_{4}\right)=1$. Later, we also consider non-Jordan domains $D$, where the boundary is to be understood in the sense of the Carathéodory boundary extension theorem. If there is no danger of confusion, we call such domains, with four points $z_{1}, z_{2}, z_{3}, z_{4}$ chosen from the Carathéodory boundary of $D$, quadrilaterals [25].

It follows immediately from the definition that the conformal modulus is invariant under conformal mappings, i.e.,

$$
\mathrm{M}\left(D ; z_{1}, z_{2}, z_{3}, z_{4}\right)=\mathrm{M}\left(f D ; f\left(z_{1}\right), f\left(z_{2}\right), f\left(z_{3}\right), f\left(z_{4}\right)\right)
$$

for any conformal mapping $f: D \rightarrow f(D)$ such that $D$ and $f(D)$ are Jordan domains.

For a curve family $\Gamma$ in the plane, we use the notation $M(\Gamma)$ for its modulus [19]. For instance, if $\Gamma$ is the family of all curves joining the opposite $a$-sides within the rectangle $[0, a] \times[0, b], a, b>0$, then $\mathrm{M}(\Gamma)=b / a$. If we consider the rectangle as a quadrilateral $Q$ with distinguished points $a+i b, i b, 0, a$, we also have $\mathrm{M}(Q ; a+i b, i b, 0, a)=b / a$; see $[1,19]$. 


\section{ETNA}

Kent State University and

Johann Radon Institute (RICAM)

Given three sets $D, E, F$, we use the notation $\Delta(E, F ; D)$ for the family of all curves joining $E$ with $F$ in $D$.

2.2. The modulus of a quadrilateral and Dirichlet integrals. One can express the modulus of a quadrilateral $\left(D ; z_{1}, z_{2}, z_{3}, z_{4}\right)$ in terms of the solution of the Dirichlet-Neumann problem as follows. Let $\gamma_{j}, j=1,2,3,4$, be the $\operatorname{arcs}$ of $\partial D$ between $\left(z_{4}, z_{1}\right),\left(z_{1}, z_{2}\right)$, $\left(z_{2}, z_{3}\right)$, and $\left(z_{3}, z_{4}\right)$, respectively. If $u$ is the (unique) harmonic solution of the DirichletNeumann problem with the boundary values of $u$ equal to 0 on $\gamma_{2}$, equal to 1 on $\gamma_{4}$, and with $\partial u / \partial n=0$ on $\gamma_{1} \cup \gamma_{3}$, then by [1, p. 65/Thm 4.5]:

$$
\mathrm{M}\left(D ; z_{1}, z_{2}, z_{3}, z_{4}\right)=\iint_{D}|\nabla u|^{2} d x d y .
$$

The function $u$ satisfying the above boundary conditions is called the potential function of the quadrilateral $\left(D ; z_{1}, z_{2}, z_{3}, z_{4}\right)$.

2.3. The modulus of a ring domain and Dirichlet integrals. Let $E$ and $F$ be two disjoint compact sets in the extended complex plane $\mathbb{C}_{\infty}$. Then one of the sets $E, F$ is bounded, and without loss of generality, we may assume that it is $E$. If both $E$ and $F$ are connected and the set $R=\mathbb{C}_{\infty} \backslash(E \cup F)$ is connected, then $R$ is called a ring domain. In this case $R$ is a doubly connected plane domain. The capacity of $R$ is defined by

$$
\operatorname{cap} R=\inf _{u} \iint_{D}|\nabla u|^{2} d x d y
$$

where the infimum is taken over all nonnegative, piecewise differentiable functions $u$ with compact support in $R \cup E$ such that $u=1$ on $E$. It is well known that there exists a unique harmonic function on $R$ with boundary values 1 on $E$ and 0 on $F$. This function is called the potential function of the ring domain $R$, and it minimizes the above integral. In other words, the minimizer may be found by solving the Dirichlet problem for the Laplace equation in $R$ with boundary values 1 on the bounded boundary component $E$ and 0 on the other boundary component $F$. A ring domain $R$ can be mapped conformally onto the annulus $\left\{z: e^{-M}<|z|<1\right\}$, where $M=\mathrm{M}(R)$ is the conformal modulus of the ring domain $R$. The modulus and capacity of a ring domain are connected by the simple identity $\mathrm{M}(R)=2 \pi / \operatorname{cap} R$. For more information on the modulus of a ring domain and its applications in complex analysis, the reader is referred to $[1,16,17,22]$.

2.4. Hyperbolic metrics. The hyperbolic geometry in the unit disk is a powerful tool of classical complex analysis. We shall now briefly review some of the main features of this geometry which are necessary for what follows. First of all, the hyperbolic distance between $x, y \in \mathbb{D}$ is given by [7]

$$
\rho_{\mathbb{D}}(x, y)=2 \operatorname{arsinh}\left(\frac{|x-y|}{\sqrt{\left(1-|x|^{2}\right)\left(1-|y|^{2}\right)}}\right) .
$$

In addition to the unit disk $\mathbb{D}$, one usually also studies the upper half plane $\mathbb{H}$ as a model of hyperbolic geometry. For $x, y \in \mathbb{H}$ we have (with $x=\left(x_{1}, x_{2}\right)$ ) [7]

$$
\rho_{\mathbb{H}}(x, y)=\operatorname{arcosh}\left(1+\frac{|x-y|^{2}}{2 x_{2} y_{2}}\right) .
$$

If there is no danger of confusion, we denote both $\rho_{\mathbb{H}}(z, w)$ and $\rho_{\mathbb{D}}(z, w)$ simply by $\rho(z, w)$. We assume that the reader is familiar with some basic facts about these geometries: geodesics, 


\section{ETNA}

Kent State University and

Johann Radon Institute (RICAM)

the hyperbolic length-minimizing curves, are circular arcs orthogonal to the boundary in each case.

Let $z_{1}, z_{2}, z_{3}, z_{4}$ be distinct points in $\mathbb{C}$. We define the absolute (cross) ratio by

$$
\left|z_{1}, z_{2}, z_{3}, z_{4}\right|=\frac{\left|z_{1}-z_{3}\right|\left|z_{2}-z_{4}\right|}{\left|z_{1}-z_{2}\right|\left|z_{3}-z_{4}\right|}
$$

This definition can be extended for $z_{1}, z_{2}, z_{3}, z_{4} \in \mathbb{C}_{\infty}$ by taking the limit. An important property of Möbius transformations is that they preserve the absolute ratios, i.e.,

$$
\left|f\left(z_{1}\right), f\left(z_{2}\right), f\left(z_{3}\right), f\left(z_{4}\right)\right|=\left|z_{1}, z_{2}, z_{3}, z_{4}\right|,
$$

if $f: \mathbb{C}_{\infty} \rightarrow \mathbb{C}_{\infty}$ is a Möbius transformation. In fact, a mapping $f: \mathbb{C}_{\infty} \rightarrow \mathbb{C}_{\infty}$ is a Möbius transformation if and only if $f$ is sense-preserving and preserves all absolute ratios.

For both $\left(\mathbb{D}, \rho_{\mathbb{D}}\right)$ and $\left(\mathbb{H}, \rho_{\mathbb{H}}\right)$ one can define the hyperbolic distance in terms of the absolute ratio. Since the absolute ratio is invariant under Möbius transformations, the hyperbolic metric also remains invariant under these transformations. In particular, any Möbius transformation of $\mathbb{D}$ onto $\mathbb{H}$ preserves the hyperbolic distances. A standard reference on hyperbolic metrics is [7].

2.5. $h \boldsymbol{p}$-FEM. In this work the natural quantity of interest is always related to the Dirichlet energy. Of course, the finite element method (FEM) is an energy-minimizing method and therefore an obvious choice. The continuous Galerkin $h p$-FEM algorithm used throughout this paper is based on our earlier work [14]. A brief outline of the relevant features used in the numerical examples below is: Babuška-Szabo-type $p$-elements, curved elements with a blending-function mapping for exact geometry, rule-based meshing for geometrically graded meshes, and in the case of an isotropic $p$ distribution, a hierarchical solution for all $p$. The main new feature considered here is the introduction of hierarchical error estimates (using auxiliary subspace techniques) for the error estimation. Hierarchical error estimates have been studied by many authors; we refer the reader to [9] and the references therein.

For the types of problems considered here, theoretically optimal conforming $h p$-adaptivity is hard. The main difficulty lies in the mesh adaptation since the desired geometry or the exponential grading is not supported by standard data structures such as Delaunay triangulations. Thus, the approach advocated here is a hybrid one, where the problem is first solved using an $a$ priori $h p$-algorithm after which the quality of the solution is estimated using error estimators specific both for the problem and the method, provided the latter are available. For instance, the exact solution or for problems concerning the conformal modulus, the so-called reciprocal error estimator are employed. The a priori algorithm is modified if the error indicators suggest modifications. If this occurs, the solution process is started anew.

In the numerical examples below, the computed results are measured with both kinds of error estimators giving us high confidence in the validity of the results and the chosen methodology.

2.5.1. Hierarchical error estimation. Consider the abstract problem setting with $V$ the standard piecewise polynomial finite element space on some discretization $T$ of the computational domain $D$. Assuming that the exact solution $u \in H_{0}^{1}(D)$ has finite energy, we arrive at the approximation problem: find $\hat{u} \in V$ such that

$$
a(\hat{u}, v)=l(v)(=a(u, v)), \quad \forall v \in V,
$$

where $a(\cdot, \cdot)$ and $l(\cdot)$ are the bilinear form and the load potential, respectively. Additional degrees of freedom can be introduced by enriching the space $V$. This is accomplished via the 


\section{ETNA}

Kent State University and

Johann Radon Institute (RICAM)

introduction of an auxiliary subspace or "error space" $W \subset H_{0}^{1}(D)$ such that $V \cap W=\{0\}$. We can then define the error problem: Find $\varepsilon \in W$ such that

$$
a(\varepsilon, v)=l(v)-a(\hat{u}, v)(=a(u-\hat{u}, v)), \quad \forall v \in W .
$$

In 2D the space $W$ that is the additional unknowns can be associated with element edges and interiors. Thus, for $h p$-methods this kind of error estimation is natural. The main result on this kind of estimators is Theorem 2.1.

THEOREM 2.1 ([12]). There is a constant $C$ depending only on the dimension $d$, the polynomial degree $p$, the continuity and coercivity constants $\mathfrak{C}$ and $\mathfrak{c}$, and the shape-regularity of the triangulation $\mathcal{T}$ such that

$$
\frac{\mathfrak{c}}{\mathfrak{C}}\|\varepsilon\|_{1} \leq\|u-\hat{u}\|_{1} \leq C\left(\|\varepsilon\|_{1}+\operatorname{osc}(R, r, \mathcal{T})\right),
$$

where the residual oscillation depends on the volumetric and face residuals $R$ and $r$ and the triangulation $\mathcal{T}$.

For this class of error estimators there are no known proofs of $p$-robustness (see [11]), but the results shown below add to the growing body of evidence that the indicator has the desired characteristics also in the standard $h p$-method setting.

The solution $\varepsilon$ of (2.1) is called the error function. It has many useful properties for both theoretical and practical considerations. In particular, the error function can be numerically evaluated and analysed for any finite element solution. This property will be used in the following. By construction the error function is identically zero at the mesh points. In Figure 4.3 one instance of a contour plot of the error function (with a detail) is displayed. This gives an excellent way to get a qualitative view of the solution which can be used to refine the discretization in the $h p$-sense. In general, evaluation of the error function requires the solution of a linear system of equations. In practice this is not an expensive computation as Theorem 2.2 indicates.

THEOREM 2.2 ([12]). The global stiffness matrix for $W$ is spectrally-equivalent to its diagonal.

Let us denote the error indicator by a pair $(e, b)$, where $e$ and $b$ refer to added polynomial degrees on edges and element interiors, respectively. It is important to notice that the estimator requires the solution of a linear system. Assuming that the enrichment is fixed over the set of $p$ problems, it is clear that the error indicator is expensive for small values of $p$ but becomes asymptotically less expensive as the value of $p$ increases. Following the recommendation of [12], our choice in the sequel is $(e, b)=(1,2)$ unless specified otherwise.

REMARK 2.3. In the case of $(0, b)$-type or pure bubble indicators, the system is not connected, and the elemental error indicators can be computed independently and thus in parallel. Therefore in practical cases one is always interested in the relative performance of $(0, b)$-type indicators.

2.6. The reciprocal identity and error estimation. Let $Q$ be a quadrilateral defined by the points $z_{1}, z_{2}, z_{3}, z_{4}$ and by boundary curves as in Section 2.1 above. The following reciprocal identity holds:

$$
\mathrm{M}\left(Q ; z_{1}, z_{2}, z_{3}, z_{4}\right) \mathrm{M}\left(Q ; z_{2}, z_{3}, z_{4}, z_{1}\right)=1 .
$$

As in $[14,15]$, we shall use the test functional

$$
\left|\mathrm{M}\left(Q ; z_{1}, z_{2}, z_{3}, z_{4}\right) \mathrm{M}\left(Q ; z_{2}, z_{3}, z_{4}, z_{1}\right)-1\right|,
$$

which by (2.2) vanishes identically, as an error estimate. 
As noted above, the error function $\varepsilon$ can be analysed in the sense of FEM-solutions. Our goal is to relate the error function given by auxiliary space techniques and the reciprocal identity arising naturally from the geometry of the problem. Let us first define the energy of the error function $\varepsilon$ as

$$
\mathcal{E}(\varepsilon)=\iint_{D}|\nabla \varepsilon|^{2} d x d y
$$

Using (2.3) the reciprocal error estimation and the error function $\varepsilon$ introduced above can be connected as follows: Let $a_{1}$ and $a_{2}$ be the moduli of the original and conjugate problems, $\varepsilon_{1}, \varepsilon_{2}$, and $\hat{\varepsilon}_{1}=\mathcal{E}\left(\varepsilon_{1}\right), \hat{\varepsilon}_{2}=\mathcal{E}\left(\varepsilon_{2}\right)$ the errors and their energies, respectively. Taking $\hat{\varepsilon}=\max \left\{\left|\hat{\varepsilon}_{1}\right|,\left|\hat{\varepsilon}_{2}\right|\right\}$ we get via direct computation:

$$
\left|1-\left(a_{1}+\hat{\varepsilon}_{1}\right)\left(a_{2}+\hat{\varepsilon}_{2}\right)\right| \leq\left|a_{1} \hat{\varepsilon}_{2}+a_{2} \hat{\varepsilon}_{1}+\hat{\varepsilon}_{1} \hat{\varepsilon}_{2}\right| \leq 2 \hat{\varepsilon} \max \left\{a_{1}, 1 / a_{1}\right\}+O\left(\hat{\varepsilon}^{2}\right) .
$$

Neglecting the higher-order term one can solve for $\hat{\varepsilon}$ and compare this with the estimates given by the individual error functions.

3. Domains with cusps. Let us recall Figure 1.2a). In general the cusps are either at the corners of enclosed regions or between two touching fibres. In terms of reference problems, the former case is idealized with hyperbolic quadrilaterals and the latter with BE-quadrilaterals (after Bergweiler and Eremenko).

3.1. Hyperbolic quadrilateral. Let $Q_{s}$ be the quadrilateral whose sides are circular arcs perpendicular to the unit circle with vertices $e^{i s}, e^{(\pi-s) i}, e^{(s-\pi) i}$, and $e^{-s i}$. We call quadrilaterals of this type hyperbolic quadrilaterals as their sides are geodesics in the hyperbolic geometry of the unit disk. We approximate the values of the modulus of $Q_{s}$.

Next we find a lower bound for the modulus of a hyperbolic quadrilateral. Let $0<\alpha<\beta<\gamma<2 \pi$. The four points $1, e^{i \alpha}, e^{i \beta}, e^{i \gamma}$ determine a hyperbolic quadrilateral whose vertices agree with these points and whose sides are orthogonal arcs terminating at these points $[14,19]$. We consider the problem of finding the modulus (or a lower bound for it) of the family $\Gamma$ of curves within the quadrilateral joining the opposite orthogonal arcs $\left(e^{i \alpha}, e^{i \beta}\right)$ and $\left(e^{i \gamma}, 1\right)$ within the quadrilateral [19]. It is easy to see that we can find a Möbius transformation $h$ of $\mathbb{D}$ onto $\mathbb{H}$ such that $h(1)=1, h\left(e^{i \alpha}\right)=t, h\left(e^{i \beta}\right)=-t, h\left(e^{i \gamma}\right)=-1$, for some $t>1$. The number $t$ can be found by setting the absolute ratios $\left|1, e^{i \alpha}, e^{i \beta}, e^{i \gamma}\right|$ and $|1, t,-t, 1|$ equal and by solving the resulting quadratic equation for $t$ because Möbius transformations preserve absolute ratios. The image quadrilateral has four semicircles as its sides, the diameters of which are $[-1,1],[1, t],[-t, t],[-t,-1]$, and the family $h(\Gamma)$ has a subfamily $\Delta$ consisting of the radial segments

$$
\left[e^{i \phi}, t e^{i \phi}\right], \quad \phi \in(\theta, \pi-\theta), \quad \sin \theta=\frac{t-1}{t+1} .
$$

Obviously, for $\theta=0$ we obtain an upper bound. Therefore

$$
\frac{\pi}{\log t} \geq \mathrm{M}(h(\Gamma)) \geq \mathrm{M}(\Delta)=\frac{\pi-2 \theta}{\log t} .
$$

3.1.1. Numerical experiments. Similarly as before, the examples of this section are outlined in Table 3.1 and Figures 3.1, 3.2. The meshes are refined in exactly the same fashion so that any differences in convergence stem only from the difference in the geometric scaling. As shown in Figure 3.3 the convergence in the reciprocal error is exponential but with a better rate for the symmetric case. Moreover, for the symmetric domain both error estimates coincide. 


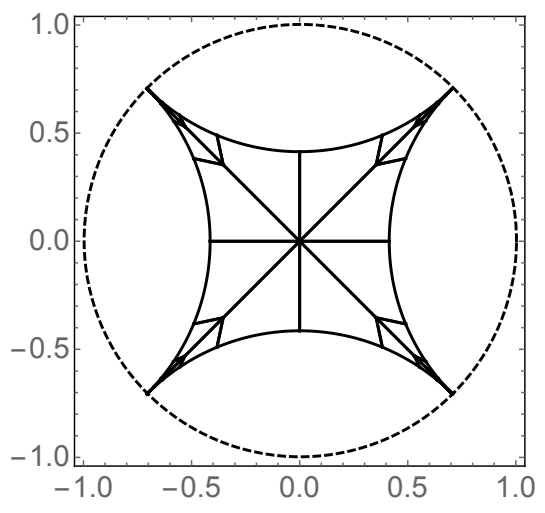

a) Case 1: $s=\pi / 4$.

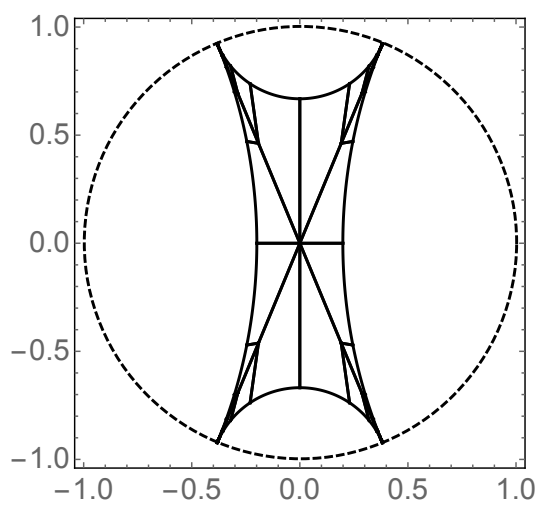

b) Case 2: $s=3 \pi / 8$.

FIG. 3.1. Hyperbolic quadrilateral: refined meshes.

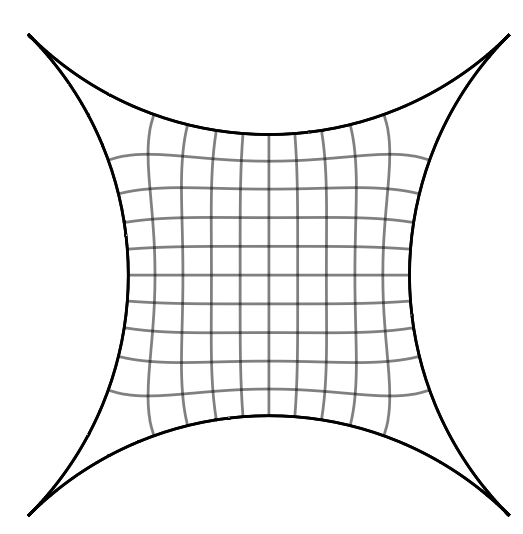

a) Case 1 .

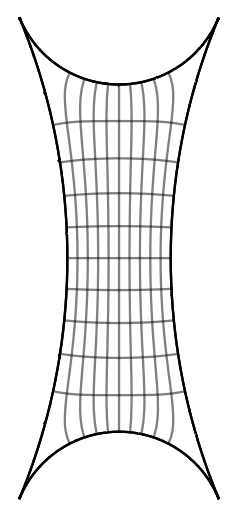

b) Case 2 .

FIG. 3.2. Hyperbolic quadrilateral: conformal maps.

3.2. Circular quadrilaterals. Above we have studied the quadrilaterals $Q=Q\left(D ; z_{1}, z_{2}, z_{3}, z_{4}\right)$ where $D$ is a Jordan domain. We next study a slight generalization where $D$ is simply connected but non-Jordan; see Section 2.1. In this case some of the points $z_{j}$ may be "double points" on the boundary. The modulus of the following quadrilateral has been obtained by W. Bergweiler and A. Eremenko [8], who studied this question in connection to an extremal problem of geometric function theory introduced by A. A. Goldberg in 1973.

3.2.1. Example I. Consider the strip from which the closed unit disk is removed:

$$
D_{1}=\{z:-3<\operatorname{Re} z<1\} \backslash \overline{\mathbb{D}} \text {. }
$$

Let the four vertices $z_{j}, j=1,2,3,4$, on the boundary of $D_{1}$ be $1, \infty, \infty, 1$, in counterclockwise order. Then, all the angles at the vertices are equal to 0 .

First we map the domain in question to a bounded domain so that the line $\{z: \operatorname{Re} z=1\}$ maps to the unit circle and the real axis remains fixed. After the Möbius transformation we may assume that we are computing in the unit disk $\mathbb{D}$. For convenience, consider the disks $\mathbb{D}(1-t, t)$ and $\mathbb{D}(-1+s, s)$ internally tangent to the unit circle at the points -1 and 1 , 


\section{ETNA}

Kent State University and

Johann Radon Institute (RICAM)

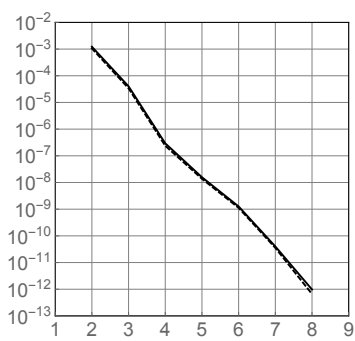

a) Case 1 .

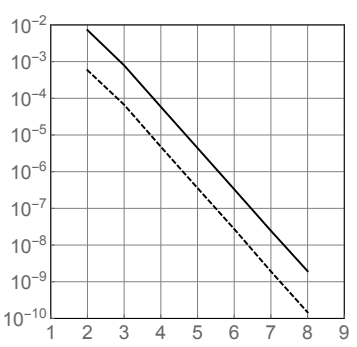

b) Case 2 .

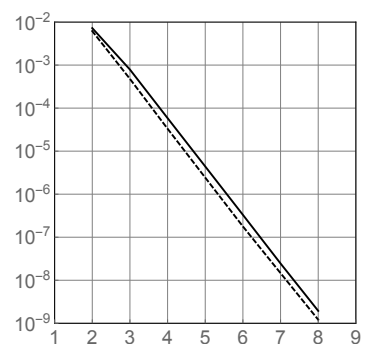

c) Case 2 (conjugate).]

FIG. 3.3. Hyperbolic quadrilateral: estimated errors; log-plot: error vs $p$; solid line $=$ reciprocal estimate, dashed line = auxiliary space estimate.

TABLE 3.1

Tests on hyperbolic quadrilaterals. The errors are given as $\mid\left\lceil\log _{10} \mid\right.$ error $\left.\mid\right\rceil \mid$.

\begin{tabular}{c|c|c|c|l} 
Case & Method & Errors & Sizes & $M\left(Q_{s}\right)$ \\
\hline 1 & $h p, p=12$ & 12 & 10225 & 1 \\
2 & $h p, p=16$ & 11 & 17985 & 3.037469188986459
\end{tabular}

respectively, with $s, t \in(0,1 / 3)$. The corner points of the quadrilateral are $1,-1,-1,1$ with a zero angle at each of the corners. We denote the respective radii of the disks by $s$ and $t$.

We have computed numerically the modulus of the family of curves joining the two disks within the domain $D_{2}=\mathbb{D} \backslash(\mathbb{D}(1-t, t) \cup \mathbb{D}(-1+s, s))$. It is the reciprocal of the modulus of the family of curves joining the upper unit semicircle with the lower unit semicircle within the same domain. The results are summarized in Table 3.2.

An estimate for the case $s=t=\sqrt{2}-1 \approx 0.41421$ is obtained by Bergweiler and Eremenko in [8] with numerical values that agree with our results up to 6 significant digits. The conformal modulus in this case is approximately 2.7823418086 ( $h p$-FEM with error number $=10, p=21$ ).

3.2.2. Example II. Consider the domain $D$ (a hexagon) in the upper half-plane obtained from the half-strip

$$
\{z=x+i y: 0<x<1,0<y\}
$$

by removing two half-disks

$$
C_{1}=\overline{\mathbb{D}(7 / 24,1 / 24)}, \quad C_{2}=\overline{\mathbb{D}(5 / 12,1 / 12)},
$$

where $\mathbb{D}(z, r)$ denotes the disk centered at $z \in \mathbb{C}$ with radius $r>0$. Note that $C_{1} \cap \mathbb{R}=[1 / 4,1 / 3]$ and $C_{2} \cap \mathbb{R}=[1 / 3,1 / 2]$. We compute the moduli of two quadrilaterals:

$$
Q_{1}=(D ; \infty, 0,1 / 2,1), \quad Q_{2}=(D ; 0,1 / 4,1 / 2,1) .
$$

Again, we first use the Möbius transformation

$$
z \mapsto \frac{2 z-1}{2 z+1}
$$

to map the domain $D$ in question to a bounded domain. Then the boundary points of $Q_{1}$ are mapped onto the points $1,-1,0,1 / 3$, respectively. For $Q_{2}$, the boundary points are 


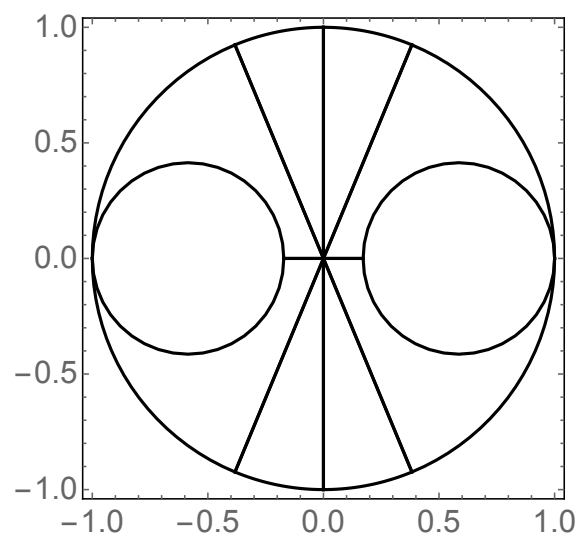

a) Case 1: $s=t=\sqrt{2}-1$.

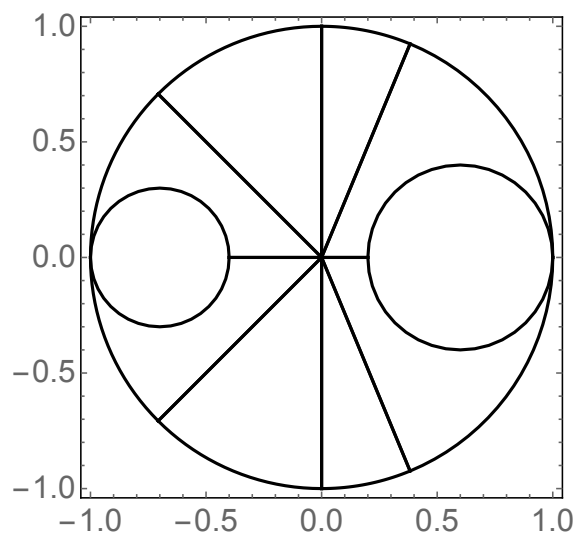

b) Case 2: $s=3 / 10, t=2 / 5$.

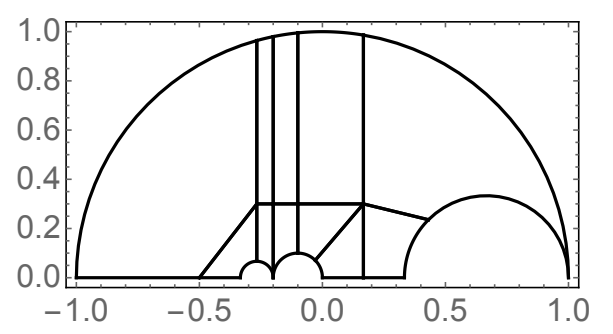

c) Case 3 and 4: $Q_{1}$ and $Q_{2}$ of example II, respectively.

FIG. 3.4. Circular quadrilaterals: p-type meshes.

TABLE 3.2

Tests on circular quadrilaterals.

\begin{tabular}{c|c|c|c|l} 
Case & Method & Errors & Sizes & $M\left(Q_{s}\right)$ \\
\hline 1 & $p, p=16$ & 9 & 1089 & 2.7823418091539533 \\
2 & $p, p=16$ & 9 & 1633 & 1.8247899464782131 \\
3 & $p, p=16$ & 8 & 2945 & 0.8852475766134157 \\
4 & $p, p=16$ & 7 & 2945 & 1.7864319361374579
\end{tabular}

mapped onto the points $-1,-1 / 3,0,1 / 3$. The quadrilaterals $Q_{1}$ and $Q_{2}$, after the Möbius transformation, and the corresponding conformal maps are illustrated in Figures 3.4c) and 3.5. The construction can easily be verified by tracing the grid lines of the maps; for instance, in Figure $3.5 \mathrm{~d})$, the boundaries $[-1,-1 / 3]$ and $[0,1 / 3]$ are clearly connected as the construction of $Q_{2}$ requires.

3.2.3. Numerical experiments. The numerical experiments differ from the previous cases since only the $p$-version is used. In other words, the meshes of Figure 3.4 are used as is without any $h$-refinement. As is evident in the convergence and error estimation graphs of Figures 3.6-3.8, in the cases where the local angles are close to $\pi / 2$, exponential convergence is achieved, but in the general case, when small geometric features are present, the convergence rates are algebraic.

4. The dendrite. A compact connected set in the plane is called dendrite-like if it contains no loops. We introduce a new parametrized family of ring domains whose boundaries 


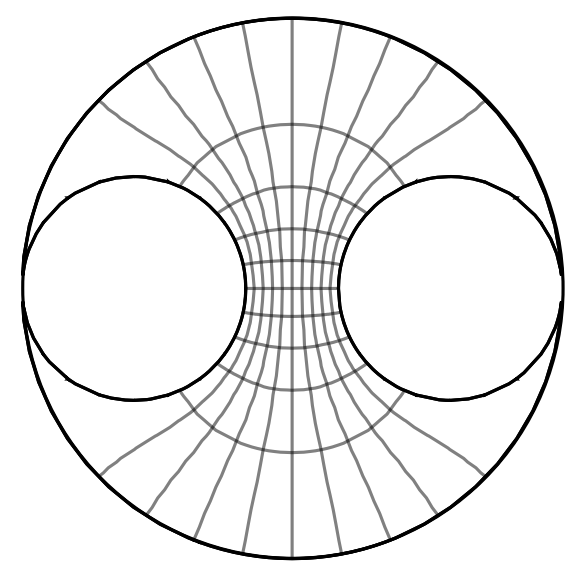

a) Case 1 .

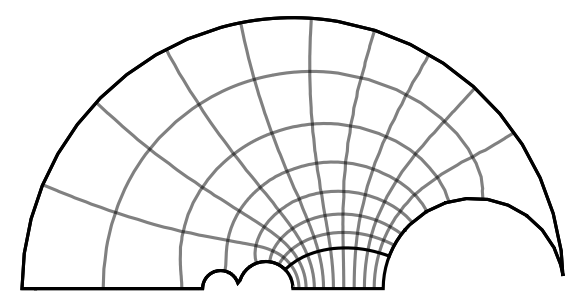

c) Case 3 .

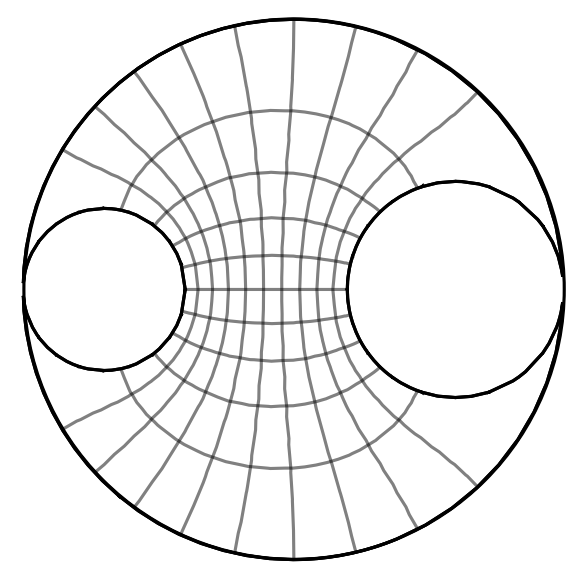

b) Case 2 .

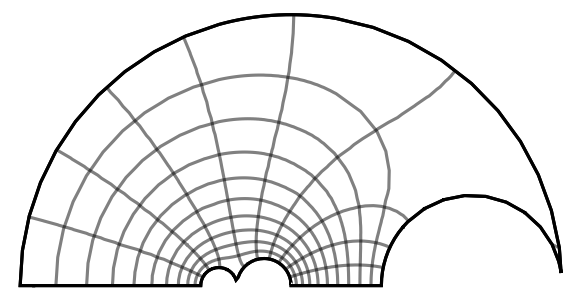

d) Case 4 .

FIG. 3.5. Circular quadrilaterals: conformal maps.

have dendrite-like boundary structure and whose modulus is explicitly known in terms of parameters. In numerical conformal mapping, one usually considers domains whose boundaries consist of finitely many piecewise smooth curves. Very recently in [23], the authors considered conformal mapping onto domains whose boundaries have "infinitely many sides", i.e., they are obtained as a result of a recursive construction. One of the examples considered in [23] was the domain whose boundary was the von Koch snowflake curve.

In this section we will give a construction of a ring domain whose complementary components are $\mathbb{C} \backslash \mathbb{D}$ and a compact connected subset $C(r, \hat{p}, m)$ of the unit disk $\mathbb{D}$ that depends on two positive integer parameters $\hat{p}, m$ and a real number $r>0$. The set $C(r, \hat{p}, m)$ is the union of finitely many pieces each of which is a smooth curve, and the set is acyclic, i.e., it does not contain any loops. The number of pieces is controlled by the integers $(\hat{p}, m)$ and can be arbitrarily large when $\hat{p}$ and $m$ increase.

4.1. Theory. Recall that the Grötzsch ring $R_{G}(r)=\mathbb{D} \backslash[0, r], r \in(0,1)$, has the capacity $\operatorname{cap}\left(R_{G}(r)\right)=2 \pi / \mu(r)$, were $\mu(r)$ is the Grötzsch modulus function (cf. [2, Chapter 5]):

$$
\mu(r)=\frac{\pi}{2} \frac{\mathcal{K}\left(r^{\prime}\right)}{\mathcal{K}(r)} \quad \text { and } \quad \mathcal{K}(r)=\int_{0}^{1} \frac{d x}{\sqrt{\left(1-x^{2}\right)\left(1-r^{2} x^{2}\right)}},
$$

with the usual notation $r^{\prime}=\sqrt{1-r^{2}}$. Let $r \in(0,1)$, and let $D_{r}=\mathbb{D} \backslash([-r, r] \cup[-i r, i r])$. The conformal mapping $f(z)=\sqrt[4]{-z}$ maps the Grötzsch ring $R_{G}(r)$ (excluding the positive real axis) onto the sector $\{z:|\arg z|<\pi / 2\}$. Let $u_{r}$ be the potential function associated with $R_{G}(r)$. Then, by symmetry, it follows that the potential function $u_{r} \circ f$ can be extended 


\section{ETNA}

Kent State University and

Johann Radon Institute (RICAM)

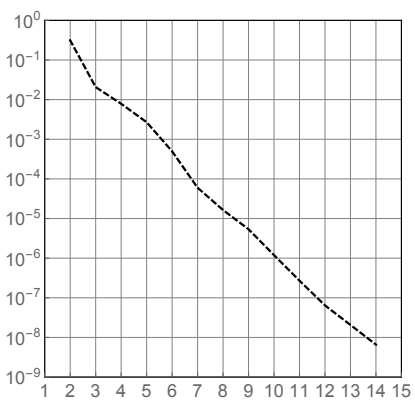

a) Reciprocal error.

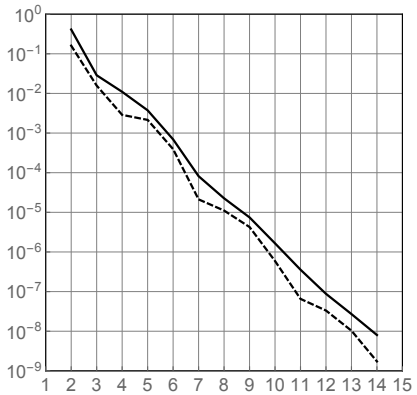

c) Estimated error (conjugate).

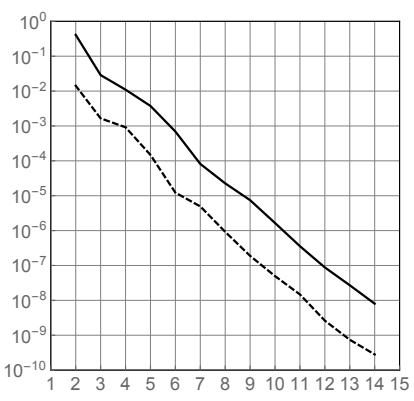

b) Estimated error.

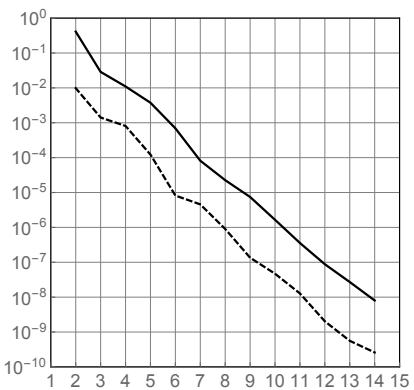

d) Estimated error using bubble-based auxiliary space: $(e, b)=(0,2)$.

FIG. 3.6. Circular quadrilaterals: comparison of error estimates: case 1: reciprocal error, log-plot: error vs p; estimated error, log-plot: error vs p; solid line = reciprocal estimate, dashed line = auxiliary space estimate.

to the domain $D_{r}$ by Schwarz symmetries so that it solves the Dirichlet problem associated with the conformal capacity of $D_{r}$. It follows that $\operatorname{cap}\left(D_{r}\right)=8 \pi / \mu\left(r^{4}\right)$. Obviously, a similar construction is possible for any integer $m \geq 3$.

One may continue the process to obtain further generalizations. Start with a generalized Grötzsch ring with $m \geq 3$ branches. Choose one of the vertices of the interior component. Map this point to the origin by a Möbius automorphism of the unit disk. Make a branch of degree $\hat{p} \geq 2$ to the origin by using the mapping $z \mapsto z^{1 / \hat{p}}$, and extend the potential function to the whole disk by using Schwarz symmetries. The resulting ring has capacity $2 \pi m \hat{p} / \mu\left(r^{m}\right)$. An example of the construction is given in Figure 4.1.

Again, it is possible to further iterate the above construction to obtain ring domains with arbitrarily complex dendrite-like boundaries. Let $m \geq 3, M \geq 1$, and let $\hat{p}_{j}$ be integers such that $\hat{p}_{j} \geq 2$, for all $j=1,2, \ldots, M$. For each $j=1,2, \ldots, M$, choose one of the vertices $z_{j}$ of the interior component. Let $w_{j}$ be the point on the line $t z_{j}, t>0$, so that $\left|w_{j}\right|=1$. We may assume that $z_{j}<0, w_{j}=-1$, and that the line segment $\left[-1, z_{j}\right]$ does not intersect with the interior component except at the point $z_{j}$. Map the point $z_{j}$ to the origin by a Möbius automorphism $g_{j}$ of the unit disk so that $g_{j}(-1)=-1$. Now map the domain $\mathbb{D} \backslash[-1,0]$ onto the symmetric disk sector by the mapping $h_{j}(z)=z^{1 / \hat{p}_{j}}$, and extend the potential function to the whole disk by using Schwarz symmetries. By repeating this construction for all $j=1,2, \ldots, M$, we obtain a ring domain with conformal capacity

$$
\frac{2 \pi m \hat{p}_{1} \hat{p}_{2} \cdots \hat{p}_{M}}{\mu\left(r^{m}\right)} .
$$




\section{ETNA}

Kent State University and

Johann Radon Institute (RICAM)

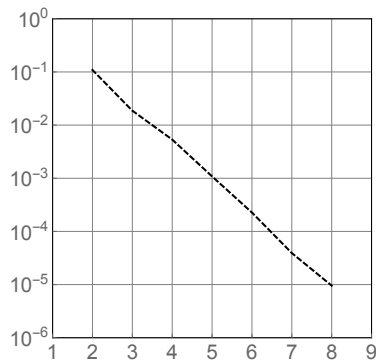

a) Case 2 .

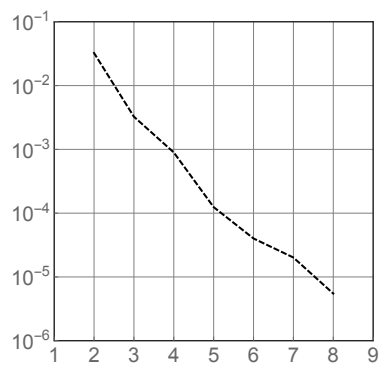

b) Case 3 .

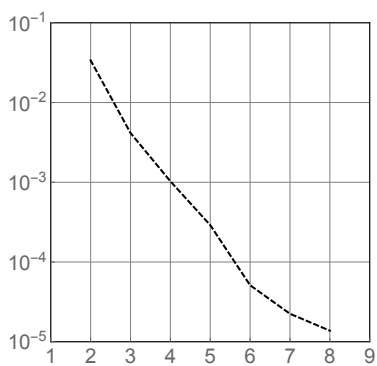

d) Case 4 .

FIG. 3.7. Circular quadrilaterals: cases 2-4: reciprocal errors; log-plot: error vs p.

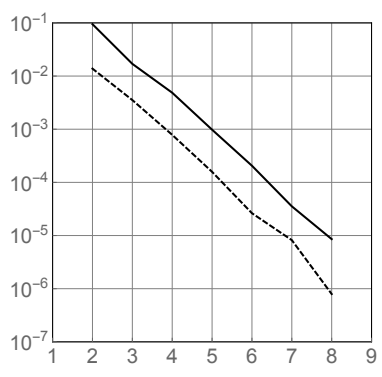

a) Case 2 .

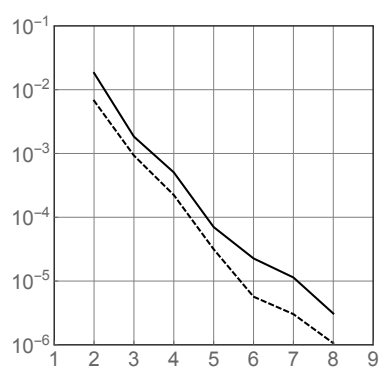

b) Case 3 .

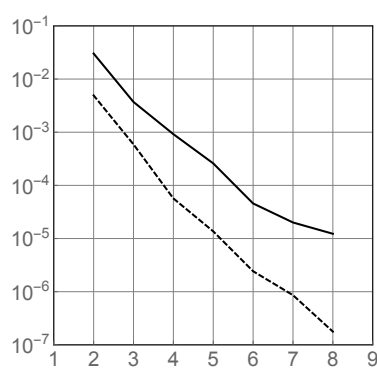

d) Case 4 .

FIG. 3.8. Circular quadrilaterals: cases 2-4: estimated errors; $\log$-plot: error vs $p$; solid line = reciprocal estimate, dashed line = auxiliary space estimate.

4.2. Numerical experiments. We consider two cases described in Table 4.1 and Figures 4.2 and 4.3. Using the $h p$-refinement strategy at the tips of the dendrite and the inner angles $\left(120^{\circ}\right.$ ), we obtain exponential convergence in the reciprocal error (Figure 4.4). In Figure 4.3 we also show the error function of type $(1,2)$ over the whole domain as well as a detail which clearly shows the non-locality of the error function. As expected, the errors are concentrated at the singularities and in the elements connecting the singularities to the boundary. One should bear in mind, however, that the reciprocal errors are small already at $p=10$ used in the figures.

The error estimates are displayed in Figure 4.5. The effect of error balancing is evident. For the larger capacity, the reciprocal error coincides with the true error but overestimates the smaller one. However, in both cases the rates are correct, only the constant is overly pessimistic. The auxiliary space error estimate underestimates the error slightly again with the correct rate.

5. Conclusions. Computational function theory contains many useful identities that are valuable also in engineering practice. In particular, the reciprocal relation is not well known outside the specialist community, yet it provides a general framework for numerical PDE-solver developers to verify codes and test discretizations in special cases.

In this paper we have introduced a new class of ring domains characterized by three parameters and given a formula for its modulus. This class of domains provides both a scalability of the computational challenge and the exactly known solution. For specific sets of parameters considered here, the convergence rates obtained for the $h p$-solution are in 


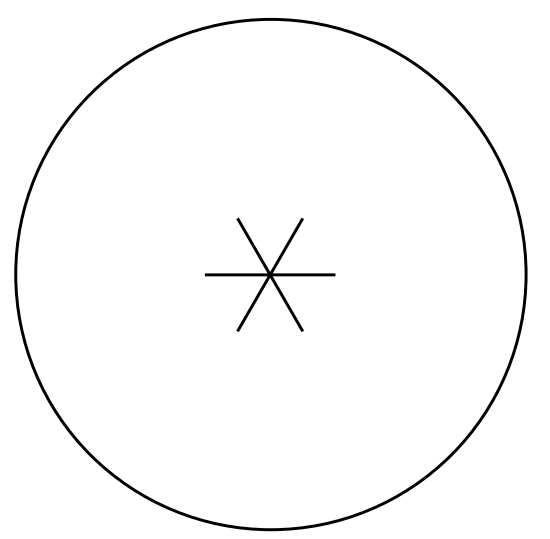

a) Generalized Grötzsch ring $R_{G}(r, m): r=1 / 4$,

$$
m=6 \text {. }
$$

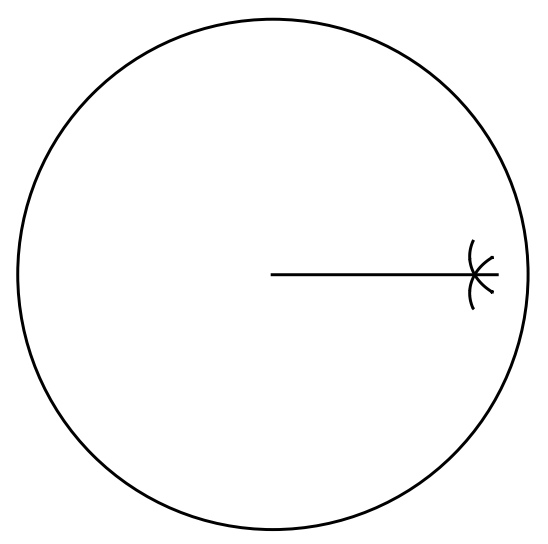

c) Make a branch of degree $p=7$.

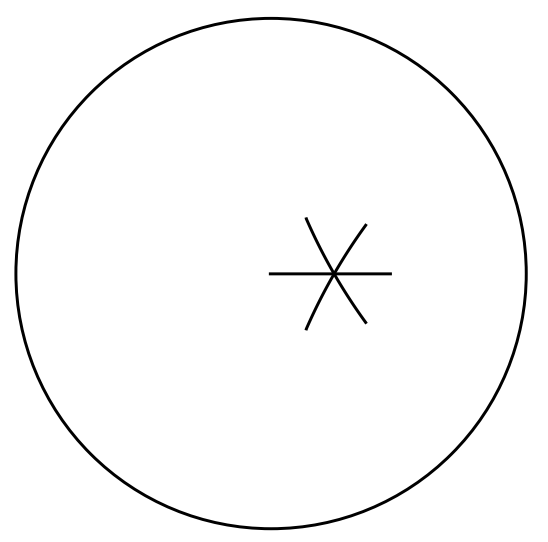

b) Map the chosen point to the origin by a Möbius automorphism of the unit disk.

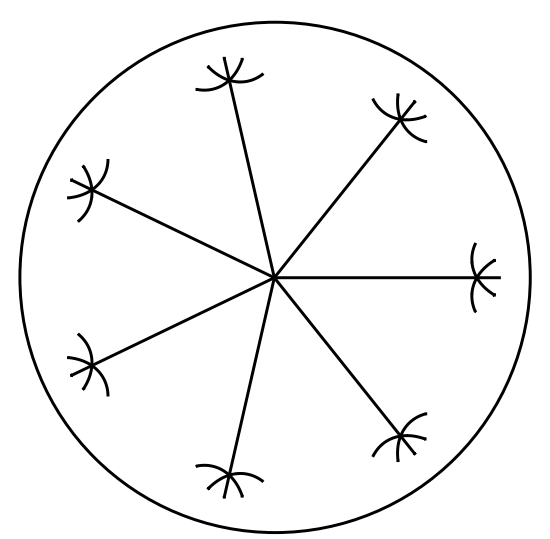

d) Extend the potential function to the whole disk.

FIG. 4.1. Dendrite Construction: $r=1 / 4, m=6, \hat{p}=7$.

accordance with theory, almost optimal, and the numerically computed error estimates behave in the same way as the true error.

Acknowledgements. This research of the third author was supported by the Academy of Finland, Project 2600066611.

\section{REFERENCES}

[1] L. V. AhLFors, Conformal Invariants: Topics in Geometric Function Theory, McGraw-Hill, New York, 1973.

[2] G. D. Anderson, M. K. Vamanamurthy, And M. Vuorinen, Conformal Invariants, Inequalities and Quasiconformal Mappings, Wiley, New York, 1997.

[3] A. Aubry, D. Y. Lei, A. Fernandez-Dominguez, Y. Sonnefraud, S. Maier, And J. B. Pendry, Plasmonic light-harvesting devices over the whole visible spectrum, Nano Lett., 10 (2010), pp. 25742579.

[4] I. BABUŠKA, X. HUANG, AND R. LIPTON, Machine computation using the exponentially convergent multiscale spectral generalized finite element method, ESAIM Math. Model. Numer. Anal., 48 (2014), pp. 493-515. 


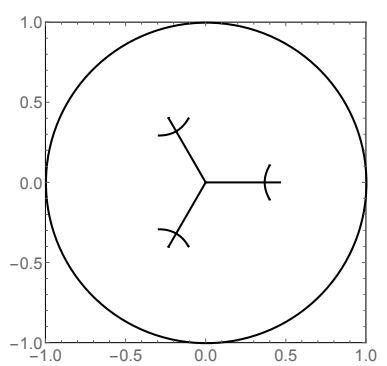

a) Domain.

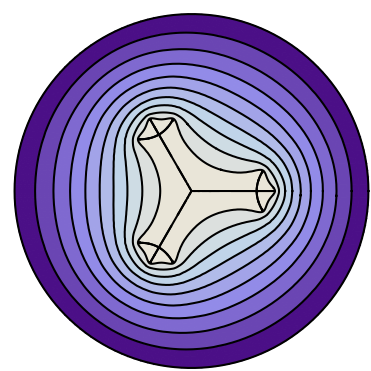

b) Potential.

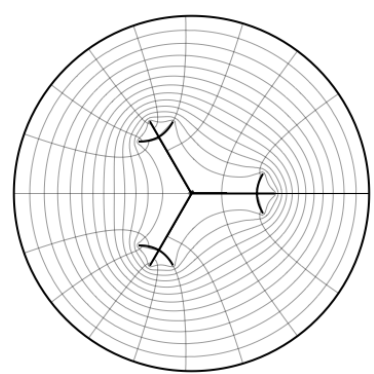

c) Conformal mapping.

FIG. 4.2. Dendrite 1: $r=1 / 20, m=4, \hat{p}=3$.

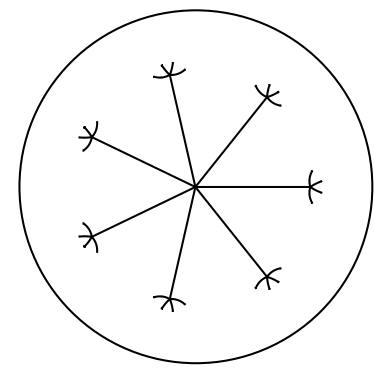

a) Domain.

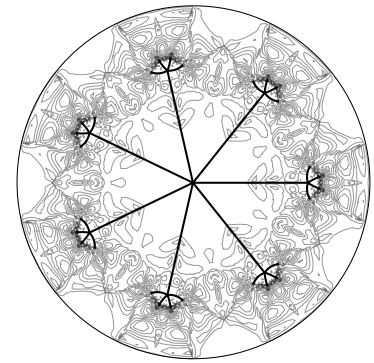

b) Contour plot: error function $(e, b)=(1,2), h p, p=10$.

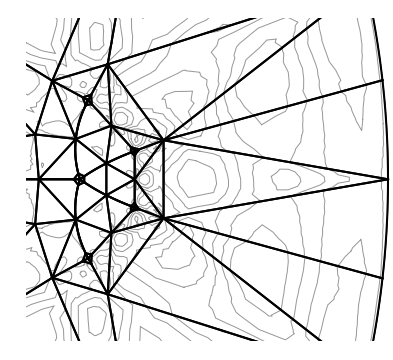

c) Contour plot: error function detail.

FIG. 4.3. Dendrite 2: $r=1 / 20, m=5, \hat{p}=7$.

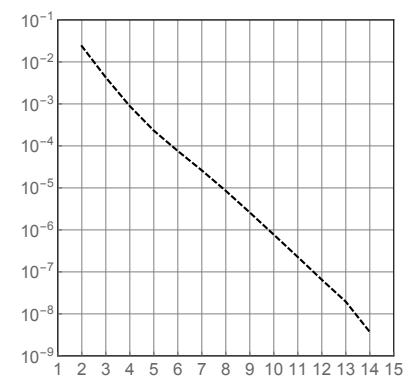

FIG. 4.4. Dendrite 1: Reciprocal error; log-plot: error vs $p$.

TABLE 4.1

Tests on dendrite-problems. The errors are given as $\mid\left\lceil\log _{10} \mid\right.$ error $\left.\mid\right\rceil \mid$.

\begin{tabular}{|c|c|c|c|c|c|}
\hline Case & Parameters & Method & Errors & Sizes & $M\left(Q_{s}\right)$ \\
\hline 1 & $\begin{array}{c}r=1 / 20 \\
m=4 \\
\hat{p}=3\end{array}$ & $h p, p=16$ & $9(9)$ & $159865(160161)$ & 5.63968609980242 \\
\hline 2 & $\begin{array}{c}r=1 / 20 \\
m=5 \\
\hat{p}=7\end{array}$ & $h p, p=12$ & $9(9)$ & 199921 (200809) & 13.437951766839522 \\
\hline
\end{tabular}




\section{ETNA}

Kent State University and

Johann Radon Institute (RICAM)

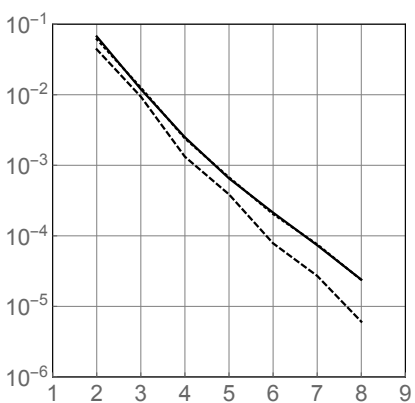

a) Estimated error.]

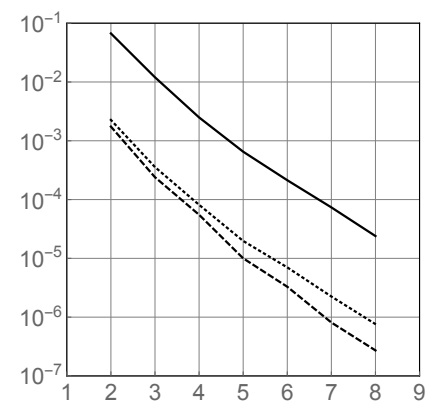

b) Estimated error (conjugate).

FIG. 4.5. Dendrite 1: estimated errors; $\log$-plot: error $v s$; solid line = reciprocal estimate, dashed line $=$ auxiliary space estimate, dotted line = exact error.

[5] L. BANJAI AND L.N. TREFETHEN, A multipole method for Schwarz-Christoffel mapping of polygons with thousands of sides, SIAM J. Sci. Comput., 25 (2003), pp. 1042-1065.

[6] M. Z. BAZANT AND D. CROWDY, Conformal mapping methods for interfacial dynamics, in Handbook of Materials Modeling, S. Yip, ed., Springer, Dordrecht, 2005, pp. 1417-1451.

[7] A. F. BEARdon, The Geometry of Discrete Groups, Springer, New York, 1983.

[8] W. Bergweiler AND A. EREmEnKo, Goldberg's constants, Anal. Math., 119 (2013), pp. 365-402.

[9] L. Demkowicz, Computing with hp-Adaptive Finite Elements. Vol. 1, Chapman \& Hall, Boca Ration, 2007.

[10] Y. DI AND R. LI, Computation of dendritic growth with level set model using a multi-mesh adaptive finite element method, J. Sci. Comput., 39 (2009), pp- 441-453.

[11] A. ERn AND M. Vohralík, Polynomial-degree-robust a posteriori estimates in a unified setting for conforming, nonconforming, discontinuous Galerkin, and mixed discretizations, SIAM J. Numer. Anal., 53 (2015), pp. 1058-1081.

[12] H. HAKUla, M. NeILAN, AND J. Ovall, A posteriori estimates using auxiliary subspace techniques, J. Sci. Comput., 72 (2017), pp. 97-127.

[13] H. Hakula, T. Quach, AND A. Rasila, Conjugate function method for numerical conformal mappings, J. Comput. Appl. Math., 237 (2013), pp. 340-353.

[14] H. HAKULA, A. RASILA, AND M. VUORINEN, On moduli of rings and quadrilaterals: algorithms and experiments, SIAM J. Sci. Comput., 33 (2011), pp. 279-302.

[15] - Computation of exterior moduli of quadrilaterals, Electron. Trans. Numer. Anal., 40 (2013), pp. 436-451.

http://etna.ricam.oeaw.ac.at/vol.40.2013/pp436-451.dir/pp436-451. pdf

[16] P. Henrici, Applied and Computational Complex Analysis. Vol. 3, Wiley, New York, 1986.

[17] R. KÜHNAU, The conformal module of quadrilaterals and of rings, in Handbook of Complex Analysis: Geometric Function Theory, R. Kühnau, ed., Elsevier, Amsterdam, 2005, pp. 99-129.

[18] R. Kyprianou, B. Yau, A. Alexopoulos, A. Verma, And B. D. Bates, Investigations into novel multi-band antenna designs, Tech. Report DSTO-TN-0719, Defence Science and Technology Organisation, Edinburgh, Australia, 2006.

[19] O. Lehto AND K. I. VirTAnen, Quasiconformal Mappings in the Plane, 2nd ed., Springer, Berlin, 1973.

[20] E. NAKOUZI AND R. SUltan, Fractal structures in two-metal electrodeposition systems I: $P b$ and Zn, Chaos, 21 (2011), Art. 043133, 8 pages.

[21] - Fractal structures in two-metal electrodeposition systems II: $\mathrm{Cu}$ and $\mathrm{Zn}$, Chaos, 22 (2012), Art. 023122, 7 pages.

[22] N. Papamichael and N. S. Stylianopoulos, Numerical Conformal Mapping: Domain Decomposition and the Mapping of Quadrilaterals, World Scientific, Hackensack, 2010.

[23] G. Riera, H. CARRASCO, AND R. PReISS, The Schwarz-Christoffel conformal mapping for polygons with infinitely many sides, Int. J. Math. Math. Sci., 2008, Art. 350326, 20 pages.

[24] R. SchinZinger And P. Laura, Conformal Mapping: Methods and Applications, Elsevier, Amsterdam, 1991. 
ETNA

Kent State University and

Johann Radon Institute (RICAM)

[25] N. SUITA, Carathéodory's theorem on boundary elements of an arbitrary plane region, Kodai Math. Sem. Rep., 21 (1969), pp. 413-417.

[26] T. TePerik, P. Nordlander, J. AizPurua, AND A. Borisov, Quantum effects and nonlocality in strongly coupled plasmonic nanowire dimers, Optics Express, 21 (2013), pp. 27306-27325.

[27] A. TIWARYA, C. HuA, AND S. GHOSH, Numerical conformal mapping method based Voronoi cell finite element model for analyzing microstructures with irregular heterogeneities, Finite Elements Anal. Design, 43 (2007), pp. 504-520.

[28] L. N. TREFETHEN AND T. A. DRISCOLL, Schwarz-Christoffel mapping in the computer era. Proceedings of the International Congress of Mathematicians, Vol. III, Doc. Math. 1998, Extra Vol. III, Deutsche Mathematiker Vereinigung, Berlin, 1998, pp. 533-542.

[29] R. WEGMANN, Methods for numerical conformal mapping, in Handbook of Complex Analysis: Geometric Function Theory. Vol. 2, R. Kühnau, ed., Elsevier, Amsterdam, 2005, pp. 351-477.

[30] G. Why burn And E. Duda, Dynamic Topology, Springer, New York, 1979. 